\title{
Mecanismos de ação de compostos bioativos dos alimentos no contexto de processos inflamatórios relacionados à obesidade
}

\author{
Effects of dietary bioactive compounds on obesity induced inflammation
}

Deborah H. M. Bastos', Marcelo M. Rogero', José Alfredo G. Arêas'

Departamento de Nutrição, Faculdade de Saúde Pública, Universidade de São Paulo (USP), São Paulo, SP, Brasil

Correspondência para: Deborah H. M. Bastos

Departamento de Nutrição,

Faculdade de Saúde Pública da Universidade de São Paulo

Av. Doutor Arnaldo, 715, 2 andar 01246-904 - São Paulo, SP, Brasil dmbastos@usp.br

Recebido em 24/Jun/2009

Aceito em 26/Jun/2009

\begin{abstract}
RESUMO
É indiscutível o papel da dieta e dos alimentos na manutenção da saúde e na redução do risco de DCNT. Estudos epidemiológicos mostram que o aumento do consumo de alimentos de origem vegetal influencia positivamente a saúde, enquanto estudos in vitro e in vivo em modelo animal elucidam os mecanismos pelos quais compostos bioativos não nutrientes, presentes nos alimentos, atuam na manutenção da saúde e na redução do risco de doenças. A modulação da expressão de genes que codificam proteínas envolvidas em vias de sinalização celular ativadas em DCNT é um dos mecanismos de ação dos compostos bioativos, sugerindo que estes possam ser essenciais à manutenção da saúde. A biodisponibilidade dos compostos bioativos de alimentos, as suas rotas metabólicas e o modo de ação de seus metabólitos são importantes fatores no seu efeito nas DCNT. Todos esses aspectos são temas de investigações recentes, cujos resultados contribuem para a compreensão da ocorrência e desenvolvimento das DCNT e da sua relação com a dieta. Essa revisão visou discutir alguns dos mecanismos envolvidos na resposta inflamatória induzida pela obesidade, apresentar os compostos bioativos de alimentos que modulam essa resposta inflamatória e sua relação com o metabolismo desses compostos. Arq Bras Endocrinol Metab. 2009;53(5):646-56.

Descritores

Inflamação; obesidade; compostos bioativos; dieta; disponibilidade biológica; doenças crônicas
\end{abstract}

\begin{abstract}
It is largely accepted the important role of food and feeding habits on health maintenance and development of non transmissible chronic diseases (NTCD). Epidemiologic evidences show that increasing vegetable consumption positively impacts health. On the other hand, in vivo and in vitro studies in animals show that non-nutrient bioactive food substances partly explain the role of food on the maintenance of health and on the risk reduction of these diseases. The modulation of gene expression of proteins that are involved in the cellular signaling pathways of NTCD is an important mechanism of the bioactive food substances, indicating their importance in disease prevention. Bioavailability, metabolic routes and the action of the resultant metabolites of bioactive food compounds are important aspects that may affect NTCD. All these aspects have actively been investigated in the last years and resulted in a greater understanding of the beginning, progression and prevention of NTCD. This review aimed at discussing the involved mechanisms of the inflammatory response induced by obesity and the role of bioactive food compounds in modulating such response. Arq Bras Endocrinol Metab. 2009;53(5):646-56.
\end{abstract}

Keywords

Inflammation; obesity; bioactive compounds; diet; biological availability; chronic disease

\section{INTRODUÇ̃̃O}

A constatação de que dietas ricas em vegetais, como Aa da população mediterrânea contemporânea e da população asiática, reduzem o risco das doenças crônicas não transmissíveis (DCNT) impulsionou pesquisas que identificaram substâncias nutrientes e não nutrientes 
atuantes em alvos fisiológicos específicos e que, dessa forma, interferem nos processos patogênicos dessas doenças. Essas evidências resultaram, entre outras coisas, em mudanças nas recomendações dos guias alimentares, os quais passaram a indicar a ingestão de maior número de porções de frutas e de hortaliças na dieta (1-4). Alimentos de origem vegetal são fontes de energia, proteína, vitaminas e minerais e a única ou principal fonte de vitamina $\mathrm{C}$, folato, fibras e compostos bioativos (CBAs), dos quais o metabolismo humano também é dependente.

Em um novo paradigma, a ingestão insuficiente de CBAs provenientes de vegetais constitui importante componente de risco das DCNT, contribuindo na mesma magnitude do consumo excessivo de energia e de gorduras totais e saturadas na dieta. Isso indica que os CBAs, da mesma forma que os demais nutrientes, são essenciais para que se atinja a carga completa (geneticamente determinada) de longevidade. Segundo esse novo paradigma, as DCNT seriam doenças relacionadas também à deficiência de substâncias "essenciais para a longevidade" (lifespan essential) (5-7).

Essa visão é complementar àquela que assume que o componente genético do homem contemporâneo está majoritariamente otimizado para a dieta de nossos ancestrais do Paleolítico. A discrepância entre a dieta contemporânea e a daquele período contribui para a etiologia das "doenças da civilização": aterosclerose, doenças cardiovasculares (DCV), resistência à insulina, diabetes melito tipo 2 (DMT2), síndrome metabólica (SM), osteoporose, hipertensão e alguns tipos de câncer (8-11). O período Paleolítico refere-se ao período da história do gênero Homo desde seu aparecimento, há mais de dois milhões de anos, até 10.000 anos, quando se iniciam a prática agrícola (predominantemente cereais) e a domesticação de animais. No período Paleolítico, que culminou com a emergência da única espécie de Homo atual, Homo sapiens, nossos ancestrais alimentavam-se de carne animal proveniente da caça (carne magra, órgãos internos, medula óssea, excluindo o leite) e plantas de ocorrência espontânea (principalmente raízes, frutos, vegetais não grãos, nozes) e outras plantas não leguminosas. Os 10.000 anos que se seguiram, quando ocorreu o desenvolvimento da agricultura e o da domesticação de animais, correspondem a $1 \%$ do tempo evolutivo do gênero Homo. Isso resultou em tempo insuficiente para que a evolução natural tivesse redesenhado o cerne metabólico e fisiológico de forma significativa para responder às mudanças de dieta introduzidas pela revolução agrícola e industrial (8-11).

Os CBAs presentes nos alimentos podem agir de diferentes formas, tanto no que se refere aos alvos fisiológicos como aos seus mecanismos de ação. A ação antioxidante, comum nesses compostos, por exemplo, deve-se ao potencial de óxido-redução de determinadas moléculas, à capacidade dessas moléculas em competir por sítios ativos e receptores nas diversas estruturas celulares ou, ainda, à modulação da expressão de genes que codificam proteínas envolvidas em mecanismos intracelulares de defesa contra processos oxidativos degenerativos de estruturas celulares (DNA, membranas).

Embora seja reconhecido que CBAs presentes na dieta atuem na manutenção da saúde, faz-se necessário reconhecer que o efeito protetor às DCNT parece não se reproduzir pela sua ingestão isolada, na forma de suplementos. Estudos clínicos, em que a dieta foi suplementada $\operatorname{com} \beta$-caroteno, vitamina $\mathrm{C}$ ou vitamina $\mathrm{E}$, mostraram que essas substâncias, isoladas da matriz alimento, não foram eficazes na diminuição de risco à DCNT (12), indicando que fatores como a biodisponibilidade e a ação sinérgica, entre outros, atuam nesse processo.

$\mathrm{Na}$ dieta habitual, alguns gramas de CBAs por dia são ingeridos. No entanto, as concentrações desses compostos no organismo humano são muito baixas - na faixa de micromoles, o que está relacionado à sua limitada absorção e biodisponibilidade.

CBAs podem ativar, por exemplo, vias de sinalização intracelulares adaptativas contra o estresse oxidativo e à exposição ao ambiente. Os CBAs exercem benefícios à saúde, ao menos em parte, atuando como "agentes de estresse de baixa dose" ou pró-oxidantes e preparando as células para resistirem às condições mais severas de estresse: doses baixas ativam vias de sinalização que resultam no aumento da expressão de genes, os quais codificam proteínas visando à proteção celular. Provavelmente, um CBA é capaz de modular uma ou duas reações in vivo que, como consequência, afetarão diferentes processos. A inibição de uma única enzima como a ciclo-oxigenase (COX-2), por exemplo, afeta a inflamação e, consequentemente, o desenvolvimento de diversas DCNT (13). Os CBAs não são denominados nutrientes, até o presente momento, por ainda não serem considerados essenciais ao crescimento e às funções vitais, conceito que, provavelmente, deverá ser revisado.

A obesidade representa grave problema de saúde pública, afetando tanto países desenvolvidos quanto em desenvolvimento. Ela está associada a um quadro 
de inflamação crônica de baixo grau, que predispõe à resistência à insulina e ao desenvolvimento do DMT2, o que implica aumento do risco para DCV. A Organização Mundial da Saúde (OMS) estimou que, no ano de 2005, havia, em todo o mundo, 400 milhões de indivíduos obesos e 1,6 bilhão de pessoas com sobrepeso, das quais 20 milhões eram crianças com idade inferior a cinco anos. A obesidade, particularmente a visceral, está intimamente relacionada à resistência periférica à insulina e ao DMT2. Essa correlação resulta no fato de que aproximadamente $30 \%$ das crianças obesas com idade inferior a 12 anos apresentem resistência periférica à insulina e que cerca de $80 \%$ dos indivíduos com DMT2 tenham sobrepeso ou obesidade (14).

Essa revisão abordou a ação de alguns CBAs vegetais na mediação de processos inflamatórios ligados à obesidade e discutiu mais aprofundadamente um importante aspecto desse processo: a biodisponibilidade.

\section{OBESIDADE E INFLAMAÇÃO}

A obesidade foi primeiramente reconhecida como uma condição de inflamação crônica de baixo grau no início da década de 1990, quando se constatou o aumento da expressão do gene que codifica para a citocina próinflamatória, denominada fator de necrose tumoral-alfa ( TNF- $\alpha$ ), no tecido adiposo e a redução da sensibilidade à insulina em roedores submetidos a um protocolo de obesidade induzida pela dieta (15). Posteriormente, outras pesquisas verificaram que a obesidade está diretamente relacionada a alterações nas funções endócrinas e metabólicas do tecido adiposo. Em indivíduos obesos, esse tecido aumenta a capacidade de síntese de moléculas com ação pró-inflamatória (denominadas adipocitocinas ou adipocinas), como a enzima óxido nítrico sintase induzível (iNOS), a proteína $\mathrm{C}$ reativa, o fator de transformação do crescimento-beta (TGF- $\beta$ ), a proteína quimiotática para monócitos (MCP-1), a molécula de adesão intracelular solúvel (sICAM), o angiotensinogênio, o inibidor-1 do ativador do plasminogênio (PAI-1), o TNF- $\alpha$, a interleucina-6 (IL-6) e a leptina $(16,17)$. A capacidade de síntese dessas proteínas, a partir do tecido adiposo de indivíduos magros, é muito inferior. Além disso, o aumento da concentração plasmática do PAI-1, o principal inibidor da fibrinólise, correlaciona-se à presença de obesidade abdominal e de outros componentes da SM. Esse biomarcador é um preditor do status da SM mais eficaz que a proteína C reativa $(17,18)$.
A resposta inflamatória promove, por um lado, o aumento da síntese de diversas adipocinas com ação pró-inflamatória e, por outro, a redução da concentração plasmática de adiponectina, que apresenta ação antiinflamatória. Esse processo reduz a expressão gênica de moléculas de adesão em células endoteliais: a liberação de TNF- $\alpha$ a partir de monócitos e a proliferação de células da musculatura lisa (16). Verifica-se forte correlação entre a redução da concentração plasmática de adiponectina e o aumento da resistência periférica à ação da insulina. A redução da gordura corporal resulta em aumento da concentração plasmática de adiponectina, em redução da resposta inflamatória e, como consequência, em diminuição da resistência periférica à ação da insulina (17).

O tecido adiposo é um tecido heterogêneo composto por adipócitos maduros e por células da fração estromal-vascular. Essa fração inclui pré-adipócitos, fibroblastos, células endoteliais, histiócitos e macrófagos. No que diz respeito à obesidade, verifica-se que o aumento de macrófagos no tecido adiposo, em particular no tecido adiposo visceral, é inicialmente precedido pelo processo de migração de monócitos do sangue para esse tecido em indivíduos obesos, cujas células, quando presentes no tecido adiposo visceral, diferenciam-se em macrófagos (18). Esse aumento do processo de quimiotaxia de monócitos, a partir do sangue para o tecido adiposo visceral, é mediado pela MCP-1, sendo que o receptor para essa proteína, denominado CCR2, é expresso em monócitos presentes no sangue periférico e em macrófagos teciduais. Além disso, a expressão da MCP-1 correlaciona-se positivamente à adiposidade, sendo a sua expressão gênica maior no tecido adiposo visceral quando comparada ao subcutâneo $(19,20)$.

O conjunto de fatores compreendendo a síntese de adipocinas, quimiocinas e citocinas; hipertrofia de adipócitos; hipoxia (deficiência de oxigênio) no tecido adiposo e endotoxemia devida ao aumento da concentração sanguínea de LPS leva a uma maior infiltração de macrófagos no tecido adiposo (14).

O recrutamento e a infiltração de macrófagos no tecido adiposo acarretam em inflamação local, que tem papel crucial no desencadeamento da resistência periférica à insulina, cuja gênese está diretamente relacionada ao aumento da concentração plasmática de diversas citocinas pró-inflamatórias, como o TNF- $\alpha$ e a IL-6 (15,20-22).

$\mathrm{O}$ TNF- $\alpha$ causa resistência à insulina por inibir a fosforilação da tirosina presente no substrato-1 do receptor de insulina (IRS-1). Outros mecanismos de inibição da fosforilação do IRS-1 por mediadores inflamatórios incluem 
a ativação crônica das proteínas Jun $\mathrm{N}$-terminal quinase (JNK), proteína quinase $\mathrm{C}(\mathrm{PKC})$ e quinase do inibidor do fator de transcrição NF- $\mathrm{KB}$ (IKK) (16-18). Além da síntese do TNF- $\alpha$, o tecido adiposo produz outras adipocinas, como a resistina, a leptina e a MCP-1, que atuam em diversas vias metabólicas, bem como na resposta inflamatória. A cultura simultânea de macrófagos e adipócitos promove a alteração da expressão da proteína transportadora de glicose 4 (GLUT4) e do IRS-1 nos adipócitos, o que pode ser parcialmente reversível pela adição de anticorpos anti-TNF- $\alpha(23)$.

O processo inflamatório estimula a diferenciação de adipócitos, o que favorece o aumento da liberação de ácidos graxos não esterificados a partir dessas células para a circulação sanguínea. Ácidos graxos não esterificados inibem o IRS-1 e, consequentemente, induzem a resistência periférica à insulina no músculo esquelético e no fígado. Entre os mecanismos associados à resistência periférica à insulina induzida por ácidos graxos não esterificados estão: (i) o estresse oxidativo, (ii) a ativação da PKC e (iii) o estresse do retículo endoplasmático $(14,17)$. O aumento do fluxo de ácidos graxos não esterificados a partir do tecido adiposo para o fígado promove resistência periférica à ação da insulina nesse tecido, devido ao aumento da expressão e atividade da enzima glicose-6-fosfatase, da gliconeogênese e da glicogenólise. Verifica-se também elevação da lipogênese e da síntese de triacilgliceróis hepática, que está relacionada à ativação do fator de transcrição denominado proteína ligadora ao elemento regulatório de esteróis (SREBP) $(17,18)$.

\section{Vias de sinalização envolvidas com a resposta inflamatória}

Entre os mecanismos moleculares envolvidos no desencadeamento da resposta inflamatória induzida pela obesidade, destaca-se a via de sinalização do fator de transcrição fator nuclear kappa B (NF- $\mathrm{BB})$, o qual aumenta a expressão de diversos genes que codificam para proteínas envolvidas na resposta inflamatória e, consequentemente, está ligado à patogênese de diferentes DCNT. A estimulação da via de sinalização do NF- $\mathrm{KB}$ pode ocorrer pela ligação de um ligante num receptor de superfície celular, como o receptor para o TNF- $\alpha$ (TNF-R) e o receptor do tipo Toll (TLR-4), que pode ser ativado tanto por lipopolissacarídeos (LPS), quanto por ácidos graxos saturados (19) (Figura 1). O aumento da ingestão de ácidos graxos saturados pela dieta favorece a ativação da resposta inflamatória e, consequentemente, o aumento do risco para DCNT (14).

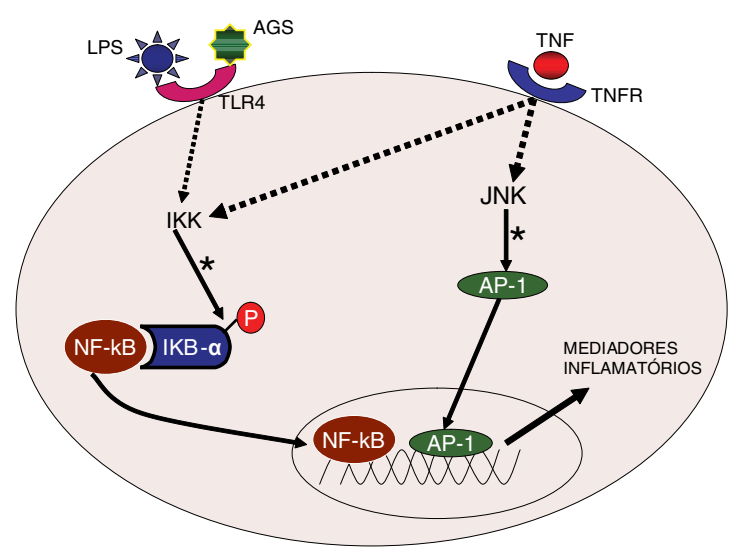

* Pontos de regulação de compostos bioativos dos alimentos.

IKB $\alpha$ : inibidor do КB; IKK: IKB quinase; TNF- $\alpha$ : fator de necrose tumoral- $\alpha$; AGS: ácidos graxos saturados; JNK: Jun N-terminal quinase; TNFR: receptor do TNF- $\alpha$; TLR-4: receptor do tipo Toll-4.

Figura 1. Vias de sinalização dos fatores de transcrição NF- $\kappa B$ e AP-1. A presença de lipopolissacarídeos (LPS) e de ácidos graxos saturados (AGS) estimula a via de sinalização do fator de transcrição denominado fator nuclear kappa B (NF-кB), enquanto a presença de TNF- $\alpha$ estimula tanto a via de sinalização do NF- $\kappa B$ quanto da proteína-1 ativadora (AP-1). A ativação dessas vias resulta no aumento da expressão de genes que codificam para proteínas envolvidas na resposta inflamatória.

Os LPS promovem a ativação de macrófagos por meio da sua ligação às proteínas CD14 e TLR4, que estão presentes na membrana plasmática. Posteriormente, há a ativação de moléculas envolvidas na transdução de sinal, como a proteína de diferenciação mieloide (MyD88), a quinase do receptor de IL-1 (IRAK), a quinase associada ao receptor do TNF (TRAF6), a quinase indutora do NF-KB (NIK) e a IKK $(16,17)$. A ativação da IKK promove a fosforilação do inibidor do fator de transcrição NF- $\kappa B(I K B-\alpha)$, o que favorece a poliubiquitinação e a subsequente degradação do IKB- $\alpha$ no proteassoma 26S, que está localizado no citosol. Subsequentemente, ocorrem a liberação e a translocação do NF- $\kappa B$ do citosol para o núcleo, onde o NF-kB (que é um fator de transcrição ubíquo, uma vez que regula a transcrição de diversos genes envolvidos nas respostas imune e inflamatória) promove a ativação de genes que codificam para proteínas envolvidas na resposta inflamatória (24).

A ativação da via de sinalização do NF- $\kappa B$ pelo TNF- $\alpha$ envolve a participação da família das proteínas quinases ativadas por mitógenos (MAPK), que atuam em reguladores upstream da via de sinalização do NF- $\kappa$ B. As MAPK representam uma família de quinases que fosforilam serina e treonina e regulam relevantes processos celulares, como crescimento, proliferação e diferenciação, por meio da modulação da transcrição gênica em resposta a alterações no ambiente intracelular. A via de sinalização da MAPK inclui quinases reguladas por sinais extracelulares (ERK- 
$1 / 2$ ), c-Jun amino-terminal quinase (JNKl $/ 2 / 3)$, p38MAP quinase $(\alpha, \beta, \delta$ e $\gamma)$ e ERK5, as quais são ativadas por específicas MAP quinase quinase (MAPKK).

As proteínas JNK e p38 são ativadas por citocinas pró-inflamatórias como TNF- $\alpha$ e IL- $1 \alpha$, bem como por outros fatores de estresse celular, como choque térmico e radiação ionizante (25). Esse fato permite referenciar essas duas quinases como "proteínas quinases ativadas pelo estresse". O aumento da atividade das MAPK e seu envolvimento na regulação da síntese de mediadores inflamatórios, tanto em nível de transcrição quanto de tradução, tornam essas enzimas alvos moleculares relevantes no contexto da nutrigenômica, inflamação e SM.

\section{MODULAÇÃO DA RESPOSTA INFLAMATÓRIA POR ÁCIDOS GRAXOS E COMPOSTOS BIOATIVOS}

\section{Ácidos graxos e resposta inflamatória}

A expansão do tecido adiposo branco é um dos modos pelos quais os ácidos graxos saturados causam alteração metabólica neste tecido. O excesso de palmitato aumenta a inflamação e a apoptose, por meio do estresse oxidativo, do estresse do retículo endoplasmático, da síntese de ceramidas e de espécies reativas de oxigênio e pela ativação da via de sinalização da PKC. Em adipócitos, o palmitato aumenta a fosforilação da JNK, ao mesmo tempo que ativa a PKC, o NFkB e a via de sinalização da MAPK, o que induz a síntese de citocinas pró-inflamatórias em adipócitos.

Ácidos graxos saturados podem causar diretamente inflamação e resistência à insulina no tecido muscular. Por exemplo, a acumulação de lipídeos no tecido, muscular mediada pelo palmitato, causa resistência periférica à insulina, por meio da via de sinalização da PKC. Adicionalmente, o palmitato aumenta a expressão e a secreção de citocinas pró-inflamatórias (IL-6 e TNF- $\alpha$ ) e prejudica a via de sinalização da insulina por meio da

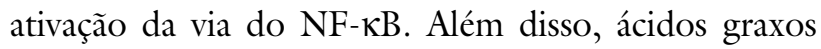
saturados induzem a resistência à insulina, por sua ação antagônica sobre o coativador do receptor ativado por proliferadores de peroxissoma gama (PPAR- $\gamma$ ) (PGC)$1 \alpha$, o qual promove a expressão de genes mitocondriais envolvidos com a fosforilação oxidativa e com a captação de glicose mediada pela insulina $(14,17,18)$.

No que concerne à relação entre inflamação e consumo de ácidos graxos trans, observa-se, a partir de estudos em humanos, que dietas ricas em ácidos graxos trans estão mais intimamente associadas à inflamação do que dietas que contêm reduzida quantidade desses áci- dos graxos. Verifica-se que indivíduos que consomem dietas ricas em ácidos graxos trans apresentam maior concentração plasmática de proteína C reativa, IL-6, E-selectina, sICAM-1 e sVCAM-1 em comparação àqueles indivíduos com reduzido consumo desses ácidos graxos. Ácidos graxos saturados têm a capacidade de ativar a via de sinalização do NF- $\mathrm{KB}$, o que aumenta a expressão de citocinas pró-inflamatórias (IL-6 e TNF- $\alpha$ ) e, consequentemente, a resistência periférica à insulina $(17,20)$.

Por outro lado, ácidos graxos poli-insaturados da série $\omega 3$, ácido eicosapentaenoico (EPA) e ácido docosaexaenoico (DHA), presentes em peixes e em óleos de peixe, apresentam ação anti-inflamatória, uma vez que diminuem a atividade dos fatores de transcrição NF- $\kappa B$ e proteína ativadora-1 (AP-1).

A superfamília de receptores nucleares é composta por fatores de transcrição que regulam positiva e negativamente a expressão gênica em resposta à ligação de diversos metabólitos e hormônios derivados de lipídeos. Entre os receptores nucleares envolvidos com o desenvolvimento da aterosclerose, destacam-se o receptor X hepático (LXR), que se subdivide em LXR $\alpha$ e LXR $\beta$, e o receptor ativado por PPAR, que se subdivide em PPAR- $\alpha$, PPAR- $\beta$ e PPAR- $\gamma$. O LXR tem papel relevante na regulação da absorção e excreção do colesterol, no efluxo celular do colesterol e no metabolismo da lipoproteína de muito baixa densidade (VLDL). Os PPAR regulam diferentes aspectos do metabolismo de lipídeos, incluindo a oxidação de ácidos graxos e o metabolismo de lipoproteínas e de glicose $(26,27)$.

A ativação do PPAR pode ocorrer por meio de ligantes oriundos da dieta, como os ácidos graxos poli-insaturados da série $\omega 3$ e os ácidos graxos monoinsaturados (ácido oleico). Entretanto, ácidos graxos saturados apresentam menor capacidade de ligação a esses fatores de transcrição, o que explica, em parte, a sua característica pró-inflamatória. A ativação do PPAR- $\alpha$ está relacionada à redução da concentração de triacilgliceróis plasmáticos, enquanto a ativação do PPAR- $\gamma$ está relacionada ao controle do metabolismo da glicose e, nesse sentido, à melhora da sensibilidade à insulina $(14,28,29)$.

\section{ALGUNS COMPOSTOS PRESENTES NOS ALIMENTOS IDENTIFICADOS COMO MODULADORES DA RESPOSTA INFLAMATÓRIA}

\section{Resveratrol e resposta inflamatória}

O resveratrol (trans-3,5,4'-triidroxiestilbeno) é uma fitoalexina composta por dois anéis fenólicos unidos por 
uma dupla ligação (Figura 2). Esse composto existe em duas isoformas: trans-resveratrol e cis-resveratrol, sendo o trans-resveratrol a forma mais estável, a qual é encontrada em uvas, bem como no vinho tinto (30).

Esse composto inibe in vitro a expressão de citocinas pró-inflamatórias em células pulmonares estimuladas com LPS e suprime a ativação dos fatores de transcrição NF- $\kappa B$ e AP-1. Similarmente, o resveratrol inibe in vitro a ativação da JNK e de sua proteína upstream denominada proteína quinase ativada por mitógenos (MEK). Este último fato pode explicar o mecanismo de supressão da ativação do fator de transcrição AP-1 pelo resveratrol.

O resveratrol também inibe in vitro a expressão gênica das enzimas COX-2 e iNOS e das moléculas de adesão de superfície celular, como a molécula-1 de adesão intercelular (ICAM-1), molécula-1 de adesão de leucócitos endotelial (ELAM-1) e molécula-1 de adesão celular vascular (VCAM-1). Uma vez que os genes que codificam para essas proteínas são regulados pelo fator de transcrição NF- $\mathrm{BB}$, é possível que esse efeito antiinflamatório do resveratrol seja decorrente da sua ação supracitada sobre a via de sinalização do NF-kB $(30,31)$.

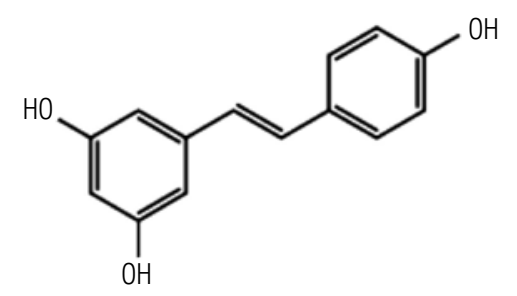

Figura 2. Estrutura do trans-resveratrol.

\section{CURCUMINA E RESPOSTA INFLAMATÓRIA}

A curcumina, um membro da família dos compostos curcuminoides, é um pigmento fenólico de cor amarela obtido a partir da cúrcuma (Curcuma longa L.), pertencente à família da Zingiberaceae. A atividade antioxidante da curcumina tem sido atribuída aos seus grupos hidroxil e metóxi (Figura 3). Um típico extrato cru dos rizomas da C. Longa contém cerca de $70 \%$ a $76 \%$ de curcumina. Diversos estudos caracterizaram a ação anti-inflamatória da curcumina, aliada à ação antibacteriana, antiviral, antifúngica e antitumoral (30).

A curcumina modula in vitro diversos alvos moleculares, incluindo o NF- $\kappa B$, e a expressão dos genes induzidos por este fator de transcrição, como as proteínas COX-2, iNOS, VCAM-1, ICAM-1, TNF- $\alpha$, IL-1, IL-6, $I L-8, I L-12$ e interferon- $\gamma$. Além disso, a curcumina ini- be in vitro a ativação do NF- $\mathrm{BB}$ dependente de TNF- $\alpha$, além da ativação induzida por forbol-12-acetato-13miristato (PMA) e por peróxido de hidrogênio. Todos esses três indutores supracitados estimulam a produção de espécies reativas de oxigênio, que são potentes ativadoras do NF- $\kappa \mathrm{B}$ (32).

Desse modo, sugere-se que seu efeito anti-inflamatório se deva, em parte, à sua capacidade de "sequestrar" espécies reativas de oxigênio em situações de estresse oxidativo celular. A curcumina é capaz de inibir a fosforilação e a degradação do IKB- $\alpha$ induzida pelo TNF- $\alpha$, o que indica que esse composto bioativo também atua em etapas que precedem a fosforilação do IKB- $\alpha$. Além disso, é capaz de inibir a ativação do fator de transcrição $\mathrm{AP}-1$, frequentemente associado à resposta inflamatória (32). Em ensaios in vitro, a curcumina efetivamente inibe a ativação da JNK em células estimuladas por TNF- $\alpha$, radiação ionizante, PMA e UV-C. Esse fato revela um dos possíveis mecanismos de supressão das vias de sinalização da AP-1 e do NF- $\kappa B$ por esse composto.

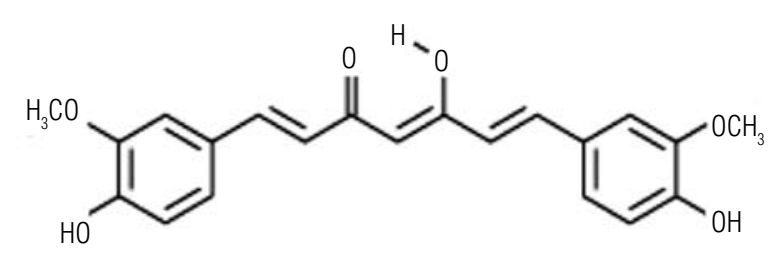

Figura 3. Estrutura da curcumina (enol).

\section{CATEQUINAS, ÁCIDOS FENÓLICOS E RESPOSTA INFLAMATÓRIA}

As catequinas são monômeros de flavanóis, como a epicatequina, a epigalocatequina, a epicatequina galato (ECG) e a epigalocatequinagalato (EGCG) (30) (Figura 4).<smiles>O=C(O[C@H]1Cc2c(O)cc(O)cc2O[C@H]1c1cc(O)c(O)c(O)c1)c1cc(O)c(O)c(O)c1</smiles>

Figura 4. Estrutura da epigalocatequinagalato.

A EGCG, o principal polifenol presente no chá verde (Camellia sinensis), tem ação anti-inflamatória, inibindo 
in vitro a ativação do fator de transcrição NF- $\kappa B$ ao mesmo tempo que inibe a degradação do IKB- $\alpha$ induzida pela ativação celular mediada pelo TNF- $\alpha$. O mecanismo de ação anti-inflamatório da EGCG parece estar associado à diminuição da atividade da proteína IKK, envolvida na fosforilação do IKB- $\alpha$. Como consequência desse efeito sobre a via de sinalização do NF- $\kappa B$, as catequinas reduzem a expressão gênica da enzima COX-2. A EGCG atua como um composto bioativo com ação anti-inflamatória na via das MAPK, inibindo a fosforilação da p38. As catequinas também reduzem a expressão gênica da proteína JNK e do fator de transcrição AP-1 $(30,31)$.

Os ácidos fenólicos, como o ácido cafeico e suas formas conjugadas (cafeoil éster) (Figura 5), compreendem uma outra família de compostos com largo espectro de atividade biológica, como a atividade anti-inflamatória, antioxidante e antiglicação, segundo os mecanismos anteriormente propostos e outros que envolvem a inibição de formação de leucotrienos - substâncias sintetizadas no metabolismo do ácido araquidônico - por meio da redução da expressão da enzima 5-lipoxigenase (5-LOX) (33-36).
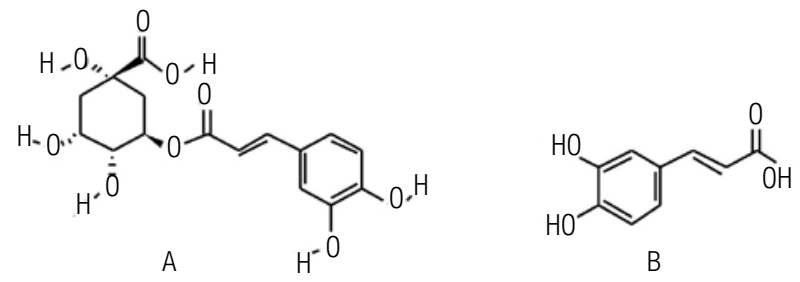

Figura 5. Estrutura do (A) ácido clorogênico (5-CQA) e (B) do ácido cafeico.

\section{QUERCETINA, TIROSOL E LICOPENO E RESPOSTA INFLAMATÓRIA}

A quercetina (frutas cítricas e maçã), o tirosol (azeite de oliva) e o licopeno (tomate, melancia e goiaba) reduzem a resposta inflamatória por meio da inibição in vitro da expressão gênica das enzimas COX-2 e iNOS, e pela diminuição da translocação do fator de transcrição NF- $\kappa B$ do citoplasma para o núcleo. A quercetina tem a capacidade de inibir as proteínas ERK e JNK e suas formas fosforiladas. Em macrófagos, a quercetina suprime a transcrição do TNF- $\alpha$ por meio da inibição da fosforilação e da ativação da JNK/SAPK, ao mesmo tempo que bloqueia a síntese de TNF- $\alpha$ por meio da inibição da fosforilação da ERK $1 / 2$ e da atividade da p38.

\section{GINGEROL, INDOL-3-CARBINOL, CAMPFEROL, APIGENINA E LUTEOLINA E A RESPOSTA INFLAMATÓRIA}

O gingerol (gengibre) e o indol-3-carbinol (crucíferas) bloqueiam a translocação da subunidade do NF- $\kappa B$ do citoplasma para o núcleo. O campferol (tomate), a apigenina (hortaliças, própolis) e a luteolina (chá, frutas e hortaliças) atuam inibindo todas as três MAPK (ERK, JNK e p38) (31).

A tabela 1 resume a relação entre os diferentes CBAs, os alimentos em que estão presentes e a ação na resposta inflamatória.

\begin{tabular}{|c|c|c|}
\hline Compostos bioativos & Fontes alimentares & Efeito na resposta inflamatória \\
\hline Resveratrol & Uvas (Vitis vinifera) & $\begin{array}{l}\text { COX-2, } \downarrow \text { iNOS, } \downarrow \text { JNK, } \downarrow \text { MEK, } \downarrow \text { NF-kappa B, } \downarrow \text { AP- } 1, \downarrow \text { PKC }, \downarrow 5-\text { LOX, } \downarrow \text { IL-6, } \downarrow \mid \mathrm{IL}-8, \downarrow \| \mathrm{IL}-1, \\
\uparrow \text { Nrf2, } \downarrow \text { VCAM- } 1\end{array}$ \\
\hline Curcumina & Cúrcuma (Curcuma longa) & $\begin{array}{l}\downarrow \text { NF-kappa B, } \downarrow \text { AP-1, } \uparrow \text { PPAR } \gamma, \uparrow \text { Nrf2, } \downarrow \text { JNK }, \downarrow \text { PKC }, \downarrow \text { VCAM-1, } \downarrow 5-\text { LOX }, \downarrow \text { COX-2, } \\
\downarrow \text { iNOS, } \downarrow \text { TNF- } \alpha, \downarrow \text { IL-6, } \downarrow \text { IL-8, } \downarrow \text { IL-12, } \uparrow \text { GSH-px }\end{array}$ \\
\hline Genisteína & Soja (Glycine max) & $\downarrow$ NF-kappa B, $\uparrow$ GSH-px \\
\hline Quercetina & Frutas cítricas, maçã & $\downarrow$ NF-kappa B \\
\hline Sulforafano & Crucíferas & $\downarrow$ NF-kappa B \\
\hline Capsaicina & Pimenta vermelha (Capsicum annum) & $\downarrow$ NF-kарра B \\
\hline Indol-3-carbinol & Crucíferas & $\downarrow$ NF-kappa B \\
\hline Ácido elágico & Romã (Punica granatum) & $\downarrow$ NF-kappa B, $\downarrow$ COX-2, $\downarrow$ MMP-9 \\
\hline 6-Gingerol & Gengibre (Zingiber officinale) & $\downarrow$ TNF- $\alpha, \downarrow$ NF-kappa B,$\downarrow$ AP- $1, \downarrow$ COX-2, $\downarrow$ iNOS, $\downarrow$ p38MAPK \\
\hline Catequinas & Chá verde (Camellia sinensis) & $\downarrow$ NF-kappa B,$\downarrow$ AP-1, $\downarrow$ JNK,$\downarrow$ COX-2, $\downarrow$ MMP-9, $\downarrow$ IL-6 \\
\hline
\end{tabular}

COX-2: ciclo-oxigenase-2; iNOS: óxido nítrico sintase induzivel; JNK: c-Jun N-terminal quinase; MEK: MAPK quinase; NF-kappa B: fator nuclear kappa B; AP-1: proteína ativadora-1; PKC: proteína quinase C; 5-LOX: 5-lipoxigenase; IL-6: interleucina-6; IL-8: interleucina-8; IL-1: interleucina-1; IL-12: interleucina-12; Nrf2: fator relacionado ao E2; VCAM-1: moléculas de adesão celular vascular-1; GSH-px: glutationa peroxidase; PPAR- $\gamma$. receptor ativado por proliferadores de peroxissomos- $\gamma$, MMP-9: metaloproteinase de matriz-9; TNF- $\alpha$ : fator de necrose tumoral. 


\section{COMPOSTOS BIOATIVOS: CONSIDERAÇÕES SOBRE SUA BIODISPONIBILIDADE}

A inconsistência de dados epidemiológicos a respeito da ingestão de chá verde e chá preto, que constituem importantes fontes de compostos fenólicos em vários países, e a redução do risco de alguns tipos de câncer são um exemplo da falta de associação entre resultados obtidos in vitro e os observados em estudos populacionais (3941). Alguns fatores podem explicar essa inconsistência:

(i) a concentração efetiva dos CBAs nos ensaios in vitro. Os estudos que avaliaram mecanismos de ação in vitro de CBAs em vias de sinalização intracelulares envolvidas na gênese de diferentes DCNT, via de regra, empregam padrões puros em concentrações muito superiores (entre dez e cem vezes) àquelas observadas in vivo após a ingestão de alimentos. Aliado a esse fato, esses estudos também não consideraram as transformações que esses CBAs sofrem durante a digestão e a absorção intestinal;

(ii) o período de exposição do "alvo biológico" aos CBAs (estudos in vitro apresentam exposição de curto-prazo, ao contrário do que ocorre in vivo);

(iii) as interações das estruturas celulares in vivo, as quais são dificilmente mantidas em estudos in vitro;

(iv) a possível interação entre os diversos compostos químicos da dieta;

(v) diferenças individuais relacionadas à constituição genética de cada indivíduo, as quais podem influenciar em todas as etapas envolvidas no estudo da biodisponibilidade de cada CBA.

A baixa biodisponibilidade de alguns CBAs, especialmente quando comparada à de macronutrientes, deve-se ao fato de o organismo reconhecer esses compostos como xenobióticos. O organismo não é capaz de distinguir compostos com potencial benéfico, tóxico ou neutro, mas somente compostos que são ou não nutrientes. Biodisponibilidade refere-se à concentração de um determinado composto ou de seus metabólitos na circulação, órgãos e tecidos em relação ao total ingerido. De maneira similar ao que ocorre com os micronutrientes, apenas uma parte dos CBAs não nutrientes é absorvido/metabolizado $(7,34)$. A absorção estimada dos polifenóis, por exemplo, situa-se entre $1 \%$ e $60 \%$ do total ingerido.

Dada a dificuldade em se analisar órgãos humanos in vivo, a determinação da biodisponibilidade de CBAs é um desafio. Em geral, os dados obtidos para os alimentos referem-se a "biodisponibilidade relativa", uma vez que a determinação da "biodisponibilidade absoluta" é realizada pela comparação entre a quantidade exata de um princípio ativo na circulação calculada como a fração da área sob a curva após a ingestão oral e aquela obtida após a administração intravenosa (7).

Muitos fatores afetam a biodisponibilidade dos CBAs, tais como: a complexidade da matriz (alimento); a forma química da substância de interesse; a estrutura e a quantidade de outros compostos presentes na dieta assim como a massa da mucosa e o tempo de trânsito intestinal; a taxa de esvaziamento gástrico; o metabolismo e o grau de conjugação e ligação com as proteínas de transporte no sangue e nos tecidos $(7,8,35,36)$. A excreção de CBAs pela via urinária, por exemplo, é maior em estudos que suplementaram a dieta com os compostos na forma isolada do que naqueles que realizaram a suplementação com a "matriz alimentar", indicando diferenças na biodisponibilidade em função da forma como o CBA está ligado ao alimento. Avaliados em conjunto, esses fatores explicam as grandes variações inter e intraindividuais relativas à biodisponibilidade de CBAs, que podem variar de $0 \%$ a $100 \%$ da dose ingerida. Assim, define-se bioacessibilidade (que constitui um pré-requisito para a biodisponibilidade) como a quantidade da substância que é potencialmente absorvível (7). Dependendo dos fatores citados anteriormente, a pouca bioacessibilidade limitará a biodisponibilidade. Os minerais, por exemplo, devem estar presentes no lúmen intestinal na forma solúvel para serem absorvidos. A formação de sais insolúveis, como quelatos com ácido fítico e oxálico, entre outros, reduz sua solubilidade e, portanto, sua biodisponibilidade.

A biodisponibilidade de CBAs durante a absorção depende das reações de biotransformações das fases I e II, características do metabolismo de xenobióticos, que ocorrem no fígado e no intestino e resultam na conjugação e/ou produção de metabólitos, que normalmente apresentam menor atividade biológica, se comparada ao composto precursor. As formas conjugadas são mais estáveis e solúveis, sendo rapidamente excretadas pelo organismo. Essas reações limitam a absorção de compostos que geralmente seriam absorvidos em razão da polaridade e da solubilidade.

CBAs não absorvidos podem apresentar atividade biológica no intestino ou estômago. Visto que o intestino é um órgão envolvido na resposta imune, a atividade local no trato digestório pode afetar a saúde de for- 
ma direta ou indireta. Ao atingir o cólon, os CBAs são hidrolisados pela microbiota originando uma gama de produtos que podem ser responsáveis pelos benefícios à saúde atribuídos aos CBAs precursores. Um exemplo desse processo é o equol, isoflavona sintetizada a partir da daidzeína (isoflavona da soja) pela microbiota do intestino, a qual apresenta atividade biológica muito superior a de seu precursor (35).

Parte dos CBAs está presente nos alimentos na forma glicosilada. No entanto, são absorvidos preferencialmente como agliconas. A hidrólise dos glicosídeos ocorre no lúmen intestinal pela ação de enzimas, como a lactase florizina hidrolase presente na membrana apical do enterócito.

Compostos fenólicos são metabólitos secundários de plantas e estão presentes em todos os vegetais consumidos como alimentos. Centenas de moléculas diferentes já foram identificadas, as quais são agrupadas em classes em função de sua estrutura química. A capacidade dessas substâncias em sequestrar radicais livres e metais pró-oxidantes explica, em parte, a correlação entre a ingestão de compostos fenólicos e a redução do risco de DCV, entre outras. Outro mecanismo responsável pela relação da ingestão de compostos fenólicos e redução de riscos de DCV refere-se à modulação gênica que interfere em diferentes processos intracelulares envolvidos na resposta inflamatória e na defesa contra o estresse oxidativo. Há várias revisões que tratam da ocorrência de compostos fenólicos, sua classificação e propriedades biológicas $(37,38)$.

Apesar dos esforços para estabelecer a relação entre a ingestão de compostos fenólicos e a redução do risco de DCNT, os principais entraves são: a) a grande diversidade de compostos fenólicos das dietas; b) a falta de dados de composição e de consumo; c) o conhecimento restrito do grau de absorção e rota metabólica de cada composto e da possível ação sinérgica ou antagonista entre eles; d) o estabelecimento de biomarcadores específicos, pois: (i) a atividade biológica observada em ensaios in vitro e em animais pode não ser similar às que ocorrem no organismo humano e (ii) compostos fenólicos diferentes podem originar um mesmo metabólito (36).

A elucidação das transformações que ocorrem com esses compostos ao longo do trato digestório, assim como nos tecidos e nos órgãos, é essencial para elucidar, por exemplo, a inconsistência de dados epidemiológicos com os dados obtidos em ensaios in vitro $\mathrm{e}$ com animais. Os polifenóis do chá verde e do chá preto sofrem ação das enzimas da fase II (como as glicosidases, sulfatases e metiltransferases) $(42,43)$ e ação do metabolismo da microbiota, processos que levam a mudanças significativas na estrutura das catequinas (conjugação ou quebra das ligações dos anéis) e, portanto, de suas propriedades físico-químicas e biológicas, conforme pode ser observado na figura $6(39)$.

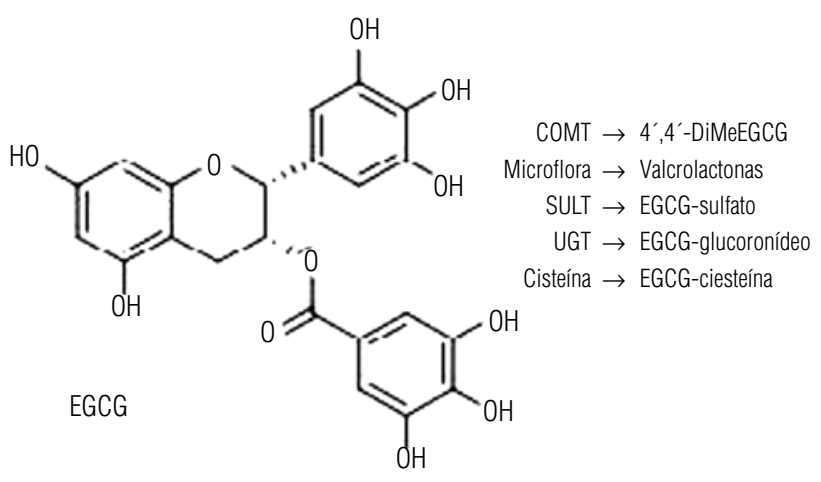

COMT: catecol-o-metil transferase; SULT: sulfotransferase; UGT,UDP: glucuroniltransferase Adaptado de Lambert e cols. (41).

Figura 6. Biotransformação da epigalocatequinagalato.

Outra classe de compostos fenólicos, amplamente difundida no reino vegetal, corresponde aos ácidos fenólicos e, entre estes, os ácidos clorogênicos, ésteres do ácido quínico com o cafeico. As propriedades biológicas dos ácidos clorogênicos (CQA) dependem da absorção intestinal e de como são metabolizados (44). Estudos com o ácido 5-cafeoilquínico (5-CQA) indicam que sua absorção começa no estômago, onde uma pequena parcela é absorvida intacta $(45,46)$. Olthof e cols. (47) demonstraram que $33 \%$ de um total de $2,8 \mathrm{mmol}$ de ácidos clorogênicos (isômeros 3,4, e 5-CQA) foram absorvidos no intestino delgado de pacientes com ileostomia. Por serem esterificados, esses compostos não são clivados no lúmen gástrico nem no intestino delgado, o que explica a baixa taxa de absorção nesses órgãos (48). A maior parte é absorvida no intestino grosso, onde ocorre hidrólise por esterases de origem microbiana e subsequente absorção dos ácidos livres (quínico, cumárico, cafeico e ferúlico) (48-50).

Durante a absorção e, posteriormente, no fígado, os ácidos clorogênicos e os ácidos fenólicos livres, deles derivados, sofrem metabolização por enzimas da fase II. Em decorrência da extensa metabolização, apenas 1\% dos ácidos clorogênicos ingeridos são encontrados intactos na urina, e os metabólitos mais abundantes são o ácido hipúrico, 3-hidroxi hipúrico e $m$-cumárico $(47,51)$. 
Estudos em animais e em humanos mostram que a curcumina apresenta baixa biodisponibilidade, independente da dose administrada. A concentração sanguínea desses compostos após a ingestão é extremamente baixa (na faixa de nanomol). Ensaios com animais determinaram que cerca de $40 \%$ a $75 \%$ da curcumina ingerida é excretada nas fezes. A presença da glucuroniltransferase e sulfotransferase (enzimas de conjugação, no fígado, rim e mucosa intestinal) sugere que a curcumina ingerida por via oral seja absorvida e circule no sangue depois de extensiva metabolização para a forma conjugada (glucuronídeos e sulfatos), apresentando rápido metabolismo e eliminação (52).

No estudo da ação do resveratrol é fortemente indicado que sejam empregados os seus metabólitos, em vez do composto na forma aglicona, como geralmente é feito em estudos in vitro que avaliam sua atividade biológica, pois esse composto também sofre transformações antes de ser absorvido e transportado para órgãos como fígado, bexiga e pulmões. As formas conjugadas do resveratrol - e não a forma livre - são preferencialmente detectadas na circulação. Deve ser observado que os alimentos considerados fonte dessa substância, como o vinho tinto, contêm outros compostos fenólicos que competirão pelas enzimas da fase II, podendo mesmo ocorrer inibição da conjugação do resveratrol, o que já foi observado em ensaios com animais (53).

\section{CONSIDERAÇÕES FINAIS}

A mudança acentuada de hábitos alimentares nas últimas décadas tem sido relacionada a muitas das DCNT, cujas prevalências aumentaram consideravelmente nos últimos tempos. As principais razões para a ocorrência desse fato encontram-se na crescente longevidade da espécie, na alimentação inadequada e no fato de o nosso organismo não estar "programado" para durar tanto tempo no estilo de vida atual, que progressivamente se tornou mais sedentário que o dos nossos antepassados. Como não se espera que seja proposto algum programa que reduza a expectativa de vida da espécie humana, só podemos modificar os outros dois fatores para reduzirmos as DCNT que contribuem negativamente para a saúde da população. Na modificação do hábito alimentar, além da adequada ingestão calórica e de nutrientes, destaca-se o aumento do consumo de frutas, hortaliças e grãos integrais. Há, atualmente, consenso sobre o fato de que essa alteração de estilo de vida, incluindo os hábitos alimentares, será capaz de reverter esse quadro que se agrava desde os meados dos anos 1970. Esta revisão focou o papel de alguns CBAs, nutrientes e não nutrientes, no processo inflamatório característico da obesidade e que é central a várias DCNT. Embora os dados da literatura sejam esparsos e incompletos, foram destacados: a biodisponibilidade de vários CBAs, seu mecanismo de ação e sua relevância no processo inflamatório. Embora o alimento não seja postulado como medicamento, acredita-se que o uso continuado de alimentos que tenham CBAs que atuam favoravelmente no processo inflamatório, aliado à manutenção de atividade física adequada, seja uma forma eficiente de reduzir o risco de aparecimento de DCNT. Apesar do grande avanço no conhecimento relativo à relação entre dieta e redução do risco de DCNT, ainda não se conhecem exatamente os mecanismos pelos quais os CBAs atuam nesse sentido. São necessárias pesquisas que identifiquem marcadores para essas substâncias e técnicas analíticas que permitam a sua quantificação.

Declaração: os autores declaram não haver conflitos de interesse científico neste estudo.

\section{REFERÊNCIAS}

1. Sabate J.The contribution of vegetarian diets to health and disease: a paradigm shift? Am J Clin Nutr. 2003;78(3 Suppl):502S-7S.

2. Larsen CS. Animal source foods and human health during evolution. J Nutr. 2003;133(11 Suppl 2):3893S-7S.

3. Minich DM, Bland JS. Dietary management of the metabolic syndrome beyond macronutrients. Nutr Rev. 2008;66(8):429-44.

4. Nestle M. Animal v. plant foods in human diets and health: is the historical record unequivocal? Proc Nutr Soc. 1999;58(2):211-8.

5. Holst B, Williamson G. Nutrients and phytochemicals: from bioavailability to bioefficacy beyond antioxidants. Curr Opin Biotechnol. 2008;19(2):73-82.

6. Williamson G, Holst B. Dietary reference intake (DRI) value for dietary polyphenols: are we heading in the right direction? $\mathrm{Br} \mathrm{J}$ Nutr. 2008;99 Suppl 3:S55-8.

7. Jacobs DR, Tapsell LC. Food, not nutrients, is the fundamental unit in nutrition. Nutr Rev. 2007;65(10):439-50.

8. Frassetto L, Morris RC, Sellmeyer DE, Todd K, Sebastian A. Diet, evolution and aging - The pathophysiologic effects of the postagricultural inversion of the potassium-to-sodium and base-tochloride ratios in the human diet. Eur J Nutr. 2001;40(5):200-13.

9. Milton K. Nutritional characteristics of wild primate foods: Do the diets of our closest living relatives have lessons for us? Nutrition. 1999;15(6):488-98.

10. Lindeberg S, Jönsson T, Granfeldt Y, Borgstrand E, Soffman J, Sjöström K, et al. A Palaeolithic diet improves glucose tolerance more than a Mediterranean-like diet in individuals with ischaemic heart disease. Diabetologia. 2007;50(9):1795-807.

11. Osterdahl M, KocturkT, Koochek A, Wandell PE. Effects of a shortterm intervention with a paleolithic diet in healthy volunteers. Eur J Clin Nutr. 2008;62(5):682-5.

12. Liu RH. Potential synergy of phytochemicals in cancer prevention: mechanism of action. J Nutr. 2004;134(12 Suppl):3479S-3485S 
13. Evans EA, Hirsch JB, Dushenkov S. Phenolics, inflammation and nutrigenomics. J Sci Food Agric. 2006;86(15):2503-2509.

14. Kennedy A, Martinez K, Chuang CC, LaPoint K, Mclntosh M. Saturated fatty acid-mediated inflammation and insulin resistance in adipose tissue: mechanisms of action and implications. J Nutr. 2009;139(1):1-4.

15. Hotamisligil GS, Shargill NS, Spiegelman BM. Adipose expression of tumor-necrosis-factor-alpha - direct role in obesity-linked insulin resistance. Science. 1993;259(5091):87-91.

16. Bullo M, Casas-Agustench $P$, Migo-Correig $P$, Aranceta J, SalasSalvado J. Inflammation, obesity and comorbidities: the role of diet. Public Health Nutr. 2007;10(10A):1164-72.

17. Shah A, Mehta N, Reilly MP. Adipose inflammation, insulin resistance, and cardiovascular disease. JPEN J Parenter Enteral Nutr. 2008;32(6):638-44.

18. Ferrante AW. Obesity-induced inflammation: a metabolic dialogue in the language of inflammation. J Intern Med. 2007;262(4):408-14.

19. Cancello R, Clément K. Is obesity an inflammatory illness? Role of low-grade inflammation and macrophage infiltration in human white adipose tissue. BJOG. 2006;113(10):1141-7.

20. Cave MC, Hurt RT, FrazierTH, Matheson PJ, Garrison RN, McClain $\mathrm{CJ}$, et al. Obesity, inflammation, and the potential application of pharmaconutrition. Nutr Clin Pract. 2008;23(1):16-34.

21. Rogero MM, Tirapegui J, Vinolo MA, Borges MC, de Castro IA, Pires IS, et al. Dietary glutamine supplementation increases the activity of peritoneal macrophages and hemopoiesis in earlyweaned mice inoculated with Mycobacterium bovis bacillus Calmette-Guérin. J Nutr. 2008;138(7):1343-8.

22. Rogero MM, Borelli $P$, Vinolo MA, Fock RA, de Oliveira Pires IS, Tirapegui J. Dietary glutamine supplementation affects macrophage function, hematopoiesis and nutritional status in early weaned mice. Clin Nutr. 2008;27(3):386-97.

23. Lumeng CN, Deyoung SM, Saltiel AR. Macrophages block insulin action in adipocytes by altering expression of signaling and glucose transport proteins. Am J Physiol Endocrinol Metab. 2007;292(1):E166-74.

24. Baud V, Karin M. OPINION: is NF-kappa B a good target for cancer therapy? Hopes and pitfalls. Nat Rev Drug Discov. 2009;8(1):33-40.

25. Rahman I, Biswas SK, Kirkham PA. Regulation of inflammation and redox signaling by dietary polyphenols. Biochem Pharmacol. 2006;72(11):1439-52.

26. Bensinger SJ, Tontonoz P. Integration of metabolism and inflammation by lipid-activated nuclear receptors. Nature. 2008;454(7203):470-7.

27. Duval C, Müller M, Kersten S. PPAR alpha and dyslipidemia. Biochim Biophys Acta. 2007;1771(8):961-71.

28. Ordovas JM. Genetic interactions with diet influence the risk of cardiovascular disease. Am J Clin Nutr. 2006;83(2):443S-446S.

29. Singh S, Aggarwal BB. Activation of transcription factor nfkappa-b is suppressed by curcumin (Diferuloylmethane). J Biol Chem. 1995;270(42):24995-25500.

30. Rahman I, Biswas SK, Kirkham PA. Regulation of inflammation and redox signaling by dietary polyphenols. Biochem Pharmacol. 2006;72(11):1439-52.

31. Aggarwal BB, Shishodia S. Molecular targets of dietary agents for prevention and therapy of cancer. Biochem Pharmacol. 2006;71(10):1397-421.

32. Hatcher H, Planalp R, Cho J, Tortia FM, Torti SV. Curcumin: From ancient medicine to current clinical trials. Cell Mol Life Sci. 2008;65(11):1631-52.

33. Góngora L, Giner RM, Máñez S, Recio MC, Schinella G, et al. Effects of caffeoyl conjugates of isoprenyl-hydroquinone glucoside and quinic acido n leukocyte function. Life Sci. 2002;71(25):2995-3004.
34. Muriel P. NF-KB in liver diseases: a target for drug therapy. J Appl Toxicol. 2009;29(2):91-100.

35. Gugliucci A, Bastos DHM. Chlorogenic acid protects oaraoxonase 1 activity in high density lipoprotein from inactivation caused by physiological concentrations of hypochlorite. Fitoterapia. 2009;80:138-142.

36. Gugliucci A, Bastos DHM, Schulze J, Souza MFF. Caffeic and chlorogenic acids in Ilex paraguariensis extracts are the main inhibitors of AGE generation by methylglyoxal in model proteins. Fitoterapia. 2009 (in press. doi:10.1016/j.fitote.2009.04.007).

37. Park HJ, Jeong SK, Kim SR, Bae SK, Kim WS, Jin SD, et al. Resveratrol inhibits Porphyromonas gingivalis lipopolysaccharideinduced endothelial adhesion molecule expression by suppressing NF-kappaB activation. Arch Pharm Res. 2009;32(4):583-91.

38. Kang SS, Cuendet M, Endringer DC, Croy VL, Pezzuto JM, Lipton MA. Synthesis and biological evaluation of a library of resveratrol analogues as inhibitors of COX-1, COX-2 and NF-kappa B. Bioorg Med Chem. 2009;17(3):1044-54.

39. Pae HO, Jeong SO, Kim HS, Kim SH, SongYS, Kim SK, et al. Dimethoxycurcumin, a synthetic curcumin analogue with higher metabolic stability, inhibits NO production, inducible NO synthase expression and NF-kappaB activation in RAW264.7 macrophages activated with LPS. Mol Nutr Food Res. 2008;52(9):1082-91.

40. Kasinski AL, DuY, Thomas SL, Zhao J, Sun SY, Khuri FR, et al. Inhibition of IkappaB kinase-nuclear factor-kappaB signaling pathway by 3,5-bis(2-flurobenzylidene)piperidin-4-one (EF24), a novel analog of curcumin. Mol Pharmacol. 2008;74(3):654-61.

41. Lambert JD, Sang SM, Yang CS. Biotransformation of green tea polyphenols and the biological activities of those metabolites. Mol Pharm. 2007;4(6):819-25.

42. Lambert JD, Yang CS. Cancer chemopreventive activity and bioavailability of tea and tea polyphenols. Mutat Res. 2003;523-524:201-8.

43. Yang CS, Maliakal P, Meng XF. Inhibition of carcinogenesis by tea. Annu Rev PharmacolToxicol. 2002;42:25-54.

44. Porrini M, Riso P. Factors influencing the bioavailability of antioxidants in foods: A critical appraisal. Nutr Metab Cardiovasc Dis. 2008;18(10):647-50.

45. Karakaya S. Bioavailability of phenolic compounds. Crit Rev Food Sci Nutr. 2004;44(6):453-64.

46. Spencer JPE, Mohsen MMA, Minihane AM, Mathers JC. Biomarkers of the intake of dietary polyphenols: strengths, limitations and application in nutrition research. Br J Nutr. 2008;99(1):12-22.

47. Olthof MR, Hollman PCH, Buijsman MNCP, van Amelsvoort JMM, Katan MB. Chlorogenic acid, quercetin-3-rutinoside and black tea phenols are extensively metabolized in humans. J Nutr. 2003;133(6):1806-14.

48. Tomas-Barberen FA, Clifford MN. Flavanones, chalcones and dihydrochalcones - nature, occurrence and dietary burden. J Sci Food Agric. 2000;80(7):1073-80.

49. Gonthier MP, Verny MA, Besson C, Remesy C, Scalbert A. Chlorogenic acid bioavailability largely depends on its metabolism by the gut microflora in rats. J Nutr. 2003;133:1853-9.

50. Clifford MN. Chlorogenic acids and other cinnamates - nature, occurrence, dietary burden, absorption and metabolism. J Sci Food Agric. 2000;80(7):1033-43.

51. Rechner AR, Spencer JPE, Kuhnle G, Hahn U, Rice-Evans CA. Novel biomarkers of the metabolism of caffeic acid derivatives in vivo. Free Radic Biol Med. 2001;30(11):1213-22.

52. Anand P, Kunnumakkara AB, Newman RA, Aggarwal BB. Bioavailability of curcumin: problems and promises. Mol Pharm. 2007;4(6):807-18.

53. Wenzel E, Somoza V. Metabolism and bioavailability of trans-resveratrol. Mol Nutr Food Res. 2005;49(5):472-81. 